\title{
Analysis of Electrooptic Modulator With 1-D Slotted Photonic Crystal Nanobeam Cavity
}

\author{
Biao Qi, Ping Yu, Yubo Li, Xiaoqing Jiang, Member, IEEE, Mei Yang, and Jianyi Yang, Member, IEEE
}

\begin{abstract}
A new nanocavity-based electrooptic modulator on silicon-on-insulator is proposed. This nanocavity is formed by a one-dimensional photonic crystal nanobeam cavity with a slot embedded. The slot lies in both the cavity and the distributed Bragg reflectors region. This results in an ultrasmall modal volume of $0.047(\lambda / n)^{3}$ and a high $Q$-factor of $8.6 \times 10^{4}$. Another advantage of the structure is that polymers can be infiltrated into the slot. Accordingly, we rely on the fast and strong electrooptic effect of polymers, rather than on the free-carrier plasma dispersion effect of silicon. Analysis shows a modulator with a bandwidth of $86 \mathrm{GHz}$, a switching voltage of $0.2 \mathrm{~V}$, and a length of only $14 \mu \mathrm{m}$ can be obtained.
\end{abstract}

Index Terms-Electrooptic effects, modulator, photonic crystal, silicon, slot.

\section{INTRODUCTION}

$\mathbf{M}$ UCH research has been carried out in the field of silicon photonics due to its potential applications in optical interconnects and its compatibility with the complementary metal-oxide-semiconductor (CMOS) technology. Electrically driven optical modulation typically relies on the free-carrier plasma dispersion effect of silicon materials. The achievable modulation speed and driving voltage are limited. The typical $V_{\pi} \cdot L$ value of silicon-based Mach-Zehnder modulators is $8 \mathrm{~V} \cdot \mathrm{cm}$ with $1 \mathrm{GHz}$ bandwidth [1]. Resonant cavity devices, such as Fabry-Pérot (FP) cavities, can generally have smaller device dimensions. Lipson's group proposed silicon photonic crystal (PhC) nanobeam cavity (NC) modulators [2], [3]. The typical modal volume of the one-dimensional (1-D) $\mathrm{PhC} \mathrm{NC} \mathrm{is}$ $0.71(\lambda / n)^{3}$ [4]. Hochberg et al. [5] proposed a silicon modulator design based on slotted waveguides [6], with which the fast and strong electrooptic (EO) effect of polymers can be applied to the silicon photonics. An experiment of $50 \mathrm{~nm}$-wide-silicon-on-insulator (SOI) slot waveguide has been reported [7]. McLauchlan et al. [8] demonstrated a silicon modulator based

Manuscript received December 09, 2010; revised March 07, 2011; accepted April 24, 2011. Date of publication April 29, 2011; date of current version July 01, 2011. This work was supported by the Major State Basic Research Development Program under Grant 2007CB307003, by the Natural Science Foundation of China under Grant 60977043, and by the Opened Fund of State Key Laboratory on Integrated Optoelectronics.

B. Qi, P. Yu, Y. Li, X. Jiang, and J. Yang are with the Department of Information Science and Electronics Engineering, Zhejiang University, Hangzhou 310027, China (e-mail: preist@zju.edu.cn; 10931018@zju.edu.cn; lilinear@zju.edu.cn; iseejxq@zju.edu.cn; yangjy@zju.edu.cn).

M. Yang is with the Department of Electrical and Computer Engineering, University of Nevada, Las Vegas, NV 89154 USA (e-mail: Mei.Yang@unlv. edu).

Color versions of one or more of the figures in this letter are available online at http://ieeexplore.ieee.org.

Digital Object Identifier 10.1109/LPT.2011.2148704

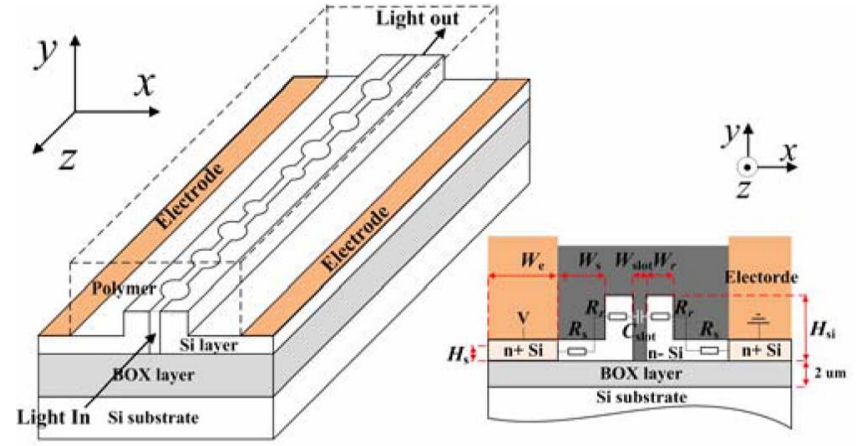

(a)

(b)

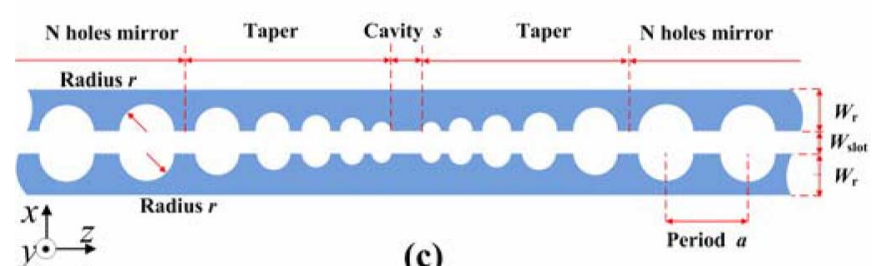

(c)

Fig. 1. (a) Schematic of the modulator with 1-D SPCNC. The slot and holes are filled with $\mathrm{EO}$ polymer in the silicon $\mathrm{PhC} \mathrm{NC}$ waveguide. The waveguide is covered with EO polymer claddings. (b) Sketch of the electrically relevant geometry of the device in the plane bisecting the cavity. (c) Top-view of the 1-D SPCNC, showing all the geometric parameters that are optimized. $N=11$.

on the 1-D FP cavities with the EO-polymer-filled slot inside the cavity region [9]. The length of the cavity is $16 \mu \mathrm{m}$ and the total device length is $31.6 \mu \mathrm{m}$. In this letter, we propose a new compact silicon modulator based on slotted photonic crystal nanobeam cavity (SPCNC) with a very low drive voltage. It is formed by a 1-D PhC NC combined with a slot embedded. The slot lies in both the cavity and the distributed Bragg reflectors region. This results in an ultrasmall cavity and modal volume. The modulation is realized by controlling the EO effect of the polymer filled in the slot and holes. The device is designed and analyzed using the three-dimensional (3-D) finite-difference time-domain (FDTD) method and the plane wave expansion method.

\section{PRINCIPLE AND ANALYSIS}

\section{A. Principle}

The configuration of the modulator with 1-D SPCNC is presented schematically in Fig. 1(a). The main idea of our approach is to utilize the phase shift induced by 1-D SPCNC which converts the phase change into light amplitude modulation. By varying the refractive index of the EO polymer, it is possible to modulate the resonance wavelength of the cavity and the intensity of light passing through the device. 
Fig. 1(b) and (c) show the schematic cross-section of the proposed configuration. A SOI $\left(n_{\mathrm{Si}}=3.47\right.$ and $\left.n_{\mathrm{SiO} 2}=1.47\right)$ slot with the width $W_{\text {slot }}$ is set along the direction parallel to the 1-D PhC NC waveguide. The original waveguide has a width of $2 W_{r}$, and the half-holes on either side of the slot are shifted outwards a distance of $W_{\text {slot }} / 2$. Hence, the width of the resulting hybrid waveguide with SPCNC is $2 W_{r}+W_{\text {slot }}$. Both the slot and holes are infiltrated with EO polymer, which can be poled with the axis of the strongest $\mathrm{EO}$ interaction aligned along the $x$-direction. In order to take advantage of the EO effect of the polymer, the proposed modulator is operated in the transverse electrical (TE)-like mode, where the optical field includes a dominant electric field component $E_{x}$. Therefore, the optical mode in the cavity is strongly constrained by both the 1-D PhC $\mathrm{NC}$ and the slot structure. This results in an ultrasmall cavity and modal volume. For comparison, the holes in the Bragg reflectors regions are not split by the slot in [8] and [9], and so the cavity length and performance are limited.

\section{B. Analysis of the Hybrid Structure With Air Claddings}

The main subject of this section is to optimize the hybrid waveguide structure to achieve high performance optical characters. Here air claddings are assumed. The silicon layer has an n-type background doping concentration of $4 \times 10^{7} \mathrm{~cm}^{-3}$, whereas highly doped $n^{+}$regions defined in the slab at each side of the ridge are considered. Ohmic contacts without additional contact resistance or capacitance have been assumed. As shown in Fig. 1(b), it is assumed that the typical value for the thickness $H_{\mathrm{si}}$ of the silicon ridge is $340 \mathrm{~nm}$, the half-width $W_{r}$. is $250 \mathrm{~nm}$, and the spacing $W_{\text {slot }}$ between the two half-ridges is $50 \mathrm{~nm}$. The choice of $H_{\mathrm{si}}$ should meet the SOI-wafer standards of the CMOS technology. The width of the highly doped regions and their distance to the ridge edges are referred to as $W_{e}=2 \mu \mathrm{m}$ and $W_{s}$, respectively. The thickness $H_{s}$ of the slab should be thin enough to provide a high lateral optical confinement and to reduce the overlap of the highly doped regions with the optical field but thick enough to facilitate its practical implementation. From the optical point of view, a slab thickness less than $50 \mathrm{~nm}$ should be desirable [2]. Here, $H_{s}=20 \mathrm{~nm}$ is assumed. It must be indicated that the slab thickness could be accurately controlled by using thermal oxidation after a ridge etching process in a practical implementation [2].

The parameters of the hybrid waveguide, including photonic mirror period $a$, holes radius $r$ as well as cavity length $s$, are optimized to achieve a good performance. As shown in Fig. 1(c), the period $a$ is linearly tapered over a five hole section from $430 \mathrm{~nm}$ to $330 \mathrm{~nm}$ at the cavity center. The hole radius is given by $r=0.28 a$. The total device length $L$ is $14 \mu \mathrm{m}$. In this case, Fig. 2 shows that the maximum $Q$-factor is above $8.4 \times 10^{4}$. A high $Q$-factor can be obtained when the resonance optical mode in the cavity perfectly match with the optical mode in the photonic mirror. As the cavity becomes longer, the resonance should red-shift due to the increase in the effective index of the cavity [4]. This trend is well consistent with our simulations. It indicates that a cavity length $s$ of $136 \mathrm{~nm}$ is required to center the resonance wavelength at $1550 \mathrm{~nm}$.

Fig. 3(a) depicts the field distribution of the dominant optical field component $E_{x}$ of the resonant mode in the $x z$ plane. It

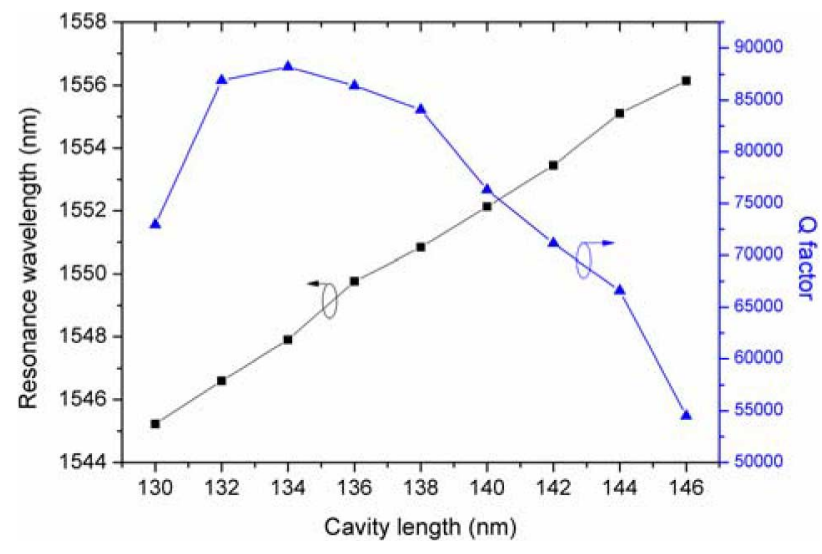

Fig. 2. Resonance wavelength and $Q$-factor as a function of the cavity length.

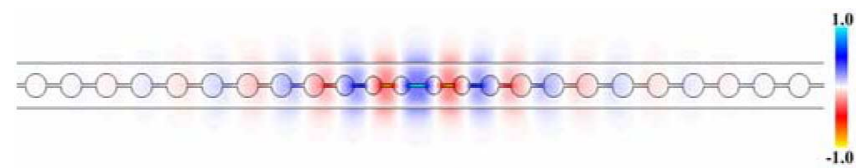

(a)

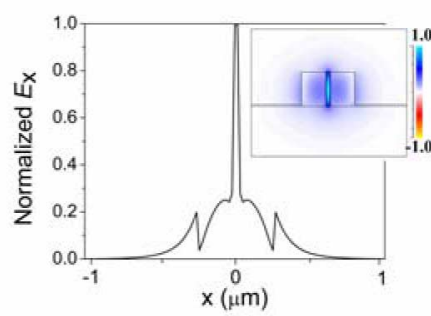

(b)

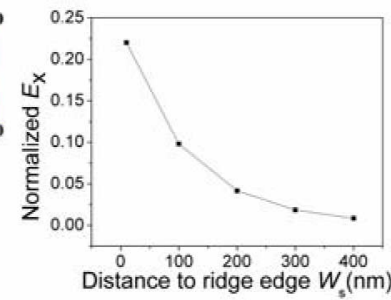

(c)
Fig. 3. (a) Profile of the resonance TE-like mode $\left(E_{x}\right)$ in the $x z$ plane. (b) Intensity profile of the resonance mode $\left(E_{x}\right)$ along the $x$-direction in the plane bisecting the cavity. Cross-section of the resonance mode $\left(E_{x}\right)$ in the cavity is shown in the inset ( $x=0$ denotes the middle of the slot layer). (c) Intensity of the resonance mode decrease as the distance to the ridge edge increases.

is revealed that a distinctive resonant mode is formed. For the TE-like optical mode, $E_{x}$ is strongly confined in the slot-layer in the cavity. The calculated $Q$ of this mode is approximately $8.6 \times 10^{4}$. It is slightly lower than the values reported for 1-D $\mathrm{PhC}$ cavities [4]. This is mostly due to the presence of the slot, and to a certain extent the slot worsens the origin performance of resonant cavities. It is proved that much higher $Q$ factors can be achieved as the slot width is carefully controlled. The calculated mode volume is $0.047(\lambda / n)^{3}$, and it is much smaller than that for 1-D and 2-D PhC cavities [4], [8].

An intensity profile of the resonant mode field $\left(E_{x}\right)$ is shown in Fig. 3(b). It is observed in the inset that the optical field shifts a little to the bottom due to the ridge slab. The distance $W_{s}$ of the highly doped regions to the ridge edges should be long enough to reduce the overlap of these highly absorptive regions with the optical field. Meanwhile, to increase of the dynamic performance, such as $3 \mathrm{~dB}$ bandwidth, of the overall device, the $W_{s}$ should be short enough. It can be pointed that the highly doped regions can be put closely to the ridge edge due to the strongly optical confinement in the SPCNC. Fig. 3(c) shows that $E_{x}$ decrease rapidly as the distance to the ridge edge increase. Following this analysis, a distance $W_{s}$ of $300 \mathrm{~nm}$ was chosen 


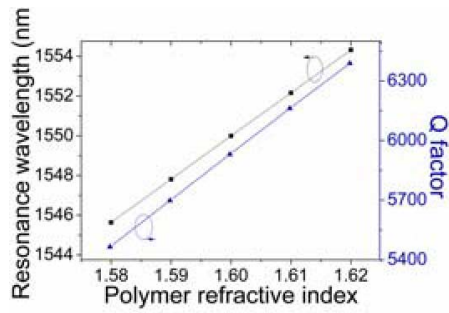

(a)

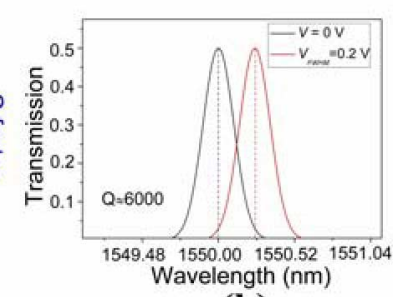

(b)
Fig. 4. (a) Resonance wavelength and $Q$-factor as a function of the refractive index in the slot and holes regions. The results are obtained by using full FDTD simulations. (b) Transmission spectrum of the cavity with 1-D SPCNC.

for the subsequent modulator design. The whole width of the device is about $5 \mu \mathrm{m}$.

\section{Analysis of the Modulator With 1-D SPCNC}

In order to make the proposed device physically realizable, more-easily-achieved polymer claddings are applied in the modulator design, as shown in Fig. 1. The similar polymer claddings of the slotted $\mathrm{PhC}$ cavity are theoretically [10] and experimentally [11] demonstrated. In this case, the period $a$ is $423 \mathrm{~nm}$ and $W_{r}$ is $225 \mathrm{~nm}$, with other parameters unchanged. A moderate $Q$ factor of about 6000 is obtained, mostly due to presence of the polymer claddings, which reduces the refractive index contrast. As depicted in Fig. 4(a), a wavelength shift of approximately $\Delta \lambda=2 \mathrm{~nm}$ can be achieved when a refractive index change of $\Delta n=0.01$ is generated. This strong sensitivity is a result of the optical field enhancement in the SPCNC region. Meanwhile, it exhibits little change in magnitude of the $Q$-factor. The change in the refractive index due to an applied electric modulation field $E$ is given by the relation $\Delta n=(1 / 2) \gamma_{33} n_{\text {poly }}^{3} E$ where $E=V / W_{\text {slot }}$. Here it is assumed that the slot width $W_{\text {slot }}$ is $50 \mathrm{~nm}$, the polymer refractive index $n_{\text {Poly }}$ is 1.6 , and the EO coefficient $\gamma_{33}$ of the polymer is $150 \mathrm{pm} / \mathrm{V}$ at the wavelength of $1550 \mathrm{~nm}$ [12]. $V$ is the applied voltage. To evaluate resonant modulator devices, the full width half maximum (FWHM) voltage $V_{\mathrm{FWHM}}$ is widely used [8], [10], which corresponds to the voltage needed to shift the resonance by its spectral width at half the maximum intensity. Using the relations given above it can be obtained that $V_{\text {FWHM }}$ is 0.2 V. Fig. 4(b) reveals the simulated transmission spectrum. The spectra shift is obtained by changing the EO polymer refractive index in the slot. As we know, the product $V \pi \cdot L$ is commonly used for the Mach-Zehnder intensity modulator [1], [13]. For comparison, the product $V \cdot L$ of the proposed modulator is $0.28 \mathrm{mV} \cdot \mathrm{cm}$, much smaller than those of the reported larger structures [1], [2], [5], [8], [10], [13].

Due to the small size, the maximum modulation frequency of the proposed 1-D SPCNC modulator is not limited by the group velocity mismatch between the electrical and optical waves. Since the device dimension is small compared to the RF wavelengths, the electrical behavior can be modeled by lumped elements [10]. The charging time is given by $\tau=2 R_{s} C_{\text {slot }}$ which yields a $3 \mathrm{~dB}$ frequency $f_{3 \mathrm{~dB}}^{R C}=1 /\left(2 \pi \times 2 R_{s} C_{\text {slot }}\right)$. As shown in Fig. 1(b), $R_{s}$ is the resistance of silicon slab between the highly doped regions and ridge edge, more than 20 times larger than the ridge resistance $R_{r}$. The polymer-filled slot regions exhibit nearly zero conductivity, which give rise to a capacitance $C_{\text {slot }}$. Assuming the resistivity of the doped silicon $\left(n_{d} \approx 4 \times 10^{17} \mathrm{~cm}^{-3}\right)$ is $\rho=0.04 \Omega \cdot \mathrm{cm}$, we obtained a large modulation frequency $f_{3 \mathrm{~dB}}$ of $86 \mathrm{GHz}$.

\section{CONCLUSION}

In summary, a compact EO modulator with 1-D SPCNC on the SOI is proposed. We come up with a new nanocavity structure, and its optical characters are analyzed. The 1-D SPCNC structure results in an ultrasmall modal volume of $0.047(\lambda / n)^{3}$ and a high $Q$-factor of $8.6 \times 10^{4}$. The EO polymers filled in the slot is employed to achieve fast and high efficient modulation. Analysis shows that the proposed device introduces improvements in terms of applied voltage and device length in a large bandwidth. Based on the proposed structure, a modulator with a bandwidth of $86 \mathrm{GHz}$, a switching voltage of $0.2 \mathrm{~V}$ and a length of only $14 \mu \mathrm{m}$ is expected.

\section{REFERENCES}

[1] A. S. Liu, R. Jones, L. Liao, D. Samara-Rubio, D. Rubin, O. Cohen, R. Nicolaescu, and M. Paniccia, "A high-speed silicon optical modulator based on a metal-oxide-semiconductor capacitor," Nature, vol. 427, pp. 615-618, 2004

[2] C. A. Barrios, V. R. Almeida, R. Panepucci, and M. Lipson, "Electrooptic modulation of silicon-on-insulator submicrometer-size waveguide devices," J. Lightw. Technol., vol. 21, no. 10, pp. 2332-2339, Oct. 2003 .

[3] B. Schmidt, Q. Xu, J. Shakya, S. Manipatruni, and M. Lipson, "Compact electro-optic modulator on silicon-on-insulator substrates using cavities with ultra-small modal volumes," Opt. Express, vol. 15, pp. 3140-3147, 2007

[4] P. B. Deotare, M. W. Mccutcheon, L. W. Frank, M. Khan, and M. Loncar, "High quality factor photonic crystal nanobeam cavities," Appl. Phys. Lett., vol. 94, p. 121106, 2009.

[5] M. Hochberg, T. Baehr-Jones, G. Wang, J. Huang, P. Sullivan, L. Dalton, and A. Scherer, "Towards a millivolt optical modulator with nano-slot waveguides," Opt. Express, vol. 15, pp. 8401-8410, 2007.

[6] V. R. Almeida, Q. Xu, C. A. Banios, and M. Lipson, "Guiding and confining light in void nanostructure," Opt. Lett., vol. 29, pp. 1209-1211, 2004.

[7] K. Foubert, L. Lalouat, B. Cluzel, E. Picard, D. Peyrade, E. Delamadeleine, F. Fornel, and E. Hadji, "Near-field modal microscopy of subwavelength light confinement in multimode silicon slot waveguides," Appl. Phys. Lett., vol. 93, p. 251103, 2008.

[8] K. K. McLauchlan and S. T. Dunham, "Analysis of a compact modulator incorporating a hybrid silicon/electro-optic polymer waveguide," IEEE J. Sel. Topics Quantum Electron., vol. 12, no. 6, pt. 2, pp. 1455-1460, Nov./Dec. 2006.

[9] J. T. Robinson, C. Manolatou, L. Chen, and M. Lipson, "Ultrasmall mode volumes in dielectric optical microcavities," Phys. Rev. Lett., vol. 95, p. 14901, 2005.

[10] J. H. Wulbern, A. Petrov, and M. Eich, "Electro-optical modulator in a polymer-infiltrated silicon slotted photonic crystal waveguide heterostucture resonator," Opt. Express, vol. 17, pp. 304-313, 2009.

[11] C. Lin, X. Wang, S. Chakravarty, B. S. Lee, W. Lai, J. Luo, A. Jen, and R. T. Chen, "Electro-optic polymer infiltrated silicon photonic crystal slot waveguide modulator with $23 \mathrm{~dB}$ slow light enhancement," Appl. Phys. Lett., vol. 97, p. 093304, 2010.

[12] T. D. Kim et al., "Ultralarge and thermally stable electrooptic activities from supramolecular selfassembled molecular glasses," J. Amer. Chem. Soc., vol. 129, pp. 488-489, 2007.

[13] B. Qi, P. Li, Y. Li, Y. L. Hao, Q. Zhou, X. Q. Jiang, and J. Y. Yang, "Ultracompact electrooptic silicon modulator with horizontal photonic crystal slotted slab," IEEE Photon. Technol. Lett., vol. 22, no. 10, pp. 724-726, May 15, 2010. 